\title{
Antibiotic treatment of streptococcal and enterococcal endocarditis: an overview
}

\author{
P. FRANCIOLI
}

\begin{abstract}
Division of Hospital Preventative Medicine and Department of Internal Medicine, Centre Hospitalier Universitaire Vaudois, 1011 Lausanne, Switzerland
\end{abstract}

KEY WORDS: Management of endocarditis, resistance.

The management of streptococcal and enterococcal endocarditis has changed in recent years with the development of effective new regimens that are easier to administer, but resistance to commonly used antibiotics has appeared, especially among enterococci. Beta-lactam antibiotics either alone or in combination are suitable for most patients with viridans streptococci. Streptococci bovis, and S. pneumoniae, but alternative regimens are necessary for special situations. Group $B, C$ and $G$ streptococci respond best to the combination of a penicillin and an aminoglycoside.

Enterococci are relatively resistant to penicillins and cephalosporins and strains resistant to beta-lactams, glycopeptides and aminoglycosides have become more common lately. Strategies are proposed dependent on the resistance of the organisms, but it is recognized that medical failure is not uncommon and surgical removal of the infected valve may be the only curative treatment.

\section{Introduction}

Streptococci account for approximately 50 to $60 \%$ of all cases of infective endocarditis, most of which are caused by viridans streptococci or Streptococcus bovis. Enterococci account for approximately $10 \%$ of cases of streptococcal endocarditis ${ }^{[1]}$. During the last few years, changes have occurred that influence the treatment of this disease ${ }^{[2]}$. New regimens that are more convenient to administer have been found to be effective, but resistance to commonly used antibiotics have appeared, especially among enterococci, that greatly compromise the success of treatment ${ }^{[3]}$. The purpose of this report is to review the present options for the antibiotic therapy of streptococcal and enterococcal endocarditis.

\section{Viridans streptococci, Streptococcus bovis}

Viridans streptococci are a heterogeneous group of microorganisms which include a variety of streptococcal species: Streptococcus sanguis, Streptococcus mitis, Streptococcus salivarius, Streptococcus mutars, Streptococcus oralis and others. Although Streptococcus bovis is a non-enterococcal group D streptococcus, its pattern of susceptibility to antibiotics is similar to that of viridans streptococci. The vast majority of these streptococci are highly sensitive to penicillin and other beta-lactam antibiotics, with minimum inhibitory concentrations of $<0.1 \mathrm{mg} . \mathrm{l}^{-1}$. However, a few strains have minimum inhibitory concentrations to penicillin greater than 1 $\mathrm{mg} . \mathrm{l}^{-1}$. Among 142 patients with streptococcal endocarditis, Wilson and Geraci observed seven such strains ${ }^{[4]}$. Some

Correspondence. Dr P. Francioli, Division of Hospital Preventative Medicine and Department of Internal Medicine, Centre Hospitalier Universitaire Vaudois, 1011 Lausanne, Switzerland. strains have minimum inhibitory concentrations to penicillin greater than $0.5 \mathrm{mg.l} \mathrm{l}^{-1}$, and therapy should be the same as that for enterococci ${ }^{[1]}$.

Although there are reports of successful treatment of streptococcal endocarditis with only a few million units of penicillin administered for a period as short as 6 days ${ }^{[5]}$, a high percentage of cures can only be obtained with prolonged therapy with high doses of antibiotics or a combination of antibiotics. There are several options for antibiotic therapy, all of which have certain advantages and disadvantages, and the selection of a specific regimen should be individualized for each patient (Tables 1 and 2).

\section{Beta-lactam therapy alone for 4 weeks}

If a beta-lactam is used alone, it should be administered for 4 weeks. In early clinical trials, penicillin at a dose of 14 to 16 million units per day and administered for 10 to 14 days still resulted in relapse rates of $15 \%$, whereas the rate for beta-lactam is only in the range of 1 to $2 \%$ when therapy is given for 4 weeks ${ }^{[4]}$.

Aqueous crystalline penicillin $\mathrm{G}$ in doses of 10 to $20 \mathrm{mil}$ lion units per day for 4 weeks is a classically recommended regimen. However, recently, ceftriaxone sodium administered at a once daily dose of $2 \mathrm{~g}$ has also been found to be an effective and safe treatment of streptococcal endocarditis $^{[26]}$. In hospital, this agent may be more convenient to administer than penicillin $\mathrm{G}$, and some patients may be able to be treated partially or entirely as outpatients. With both of these agents, bacteriological cure can be expected in approximately $99 \%$ of the patients ${ }^{[7]}$.

\section{Beta-lactam for 2 weeks + aminoglycoside for 2 weeks}

Because of evidence in-vitro and from animal models of synergism between beta-lactams and aminoglycosides, shorter courses of combined therapy have been tested. Although older reports of the combination of penicillin 
Table I Therapeutic options in streptococcal endocarditis

(A) Viridans streptococci, $S$ bovis ( 2 minımum inhibitory concentrations $<0.1 \mathrm{mg} . \mathrm{l}^{-1}$

1. Beta-lactam therapy alone $\times 4$ weeks

2. Vancomycin or teicoplanin $\times 4$ weeks

3. Beta-lactam $\times 2$ weeks + aminoglycoside $\times 2$ weeks

4. Combined parenteral and oral regimens

5. Outpatient therapy options

(B) Particular cases

Nutritionally deficient variants of viridans streptococci

Minimum inhibitory concentration $>0.1 \mathrm{mg} .1^{-1}<0.5 \mathrm{mg} .1^{-1}$

Duration of symptoms $>3$ months

Extracardiac infection, mycotic aneurysm

1. Beta-lactam $\times 4$ weeks + aminoglycoside $\times 2-4$ weeks

2. Vancomycin $\times 4$ weeks + aminoglycoside $\times 2-4$ weeks

(C) S pneumoniae, group A streptococci

1. Beta-lactam $\times 4$ weeks

2. Vancomycin $\times 4$ weeks

(D) Group B, C, G streptococci

1. Beta-lactam $\times 4$ weeks + aminoglycoside $\times 2$ weeks

2. Vancomycin $\times 4$ weeks + aminogylcoside $\times 2$ weeks

and streptomycin indicated failure rates in the range of 6 to $11 \%$, more recent reports have shown excellent results, with bacteriological cure rates as high as $99 \%$ in selected cases $^{|8|}$. Recently, a 14-day course of ceftriaxone combined with netilmicin, both administered as a single daily dose, has been tested in a multicentre trial. Among 43 bacteriologically assessable cases, only one relapse occurred in a case caused by Gemella morbillorum, a microorganism which has been associated with an unusual course of endocarditis $^{[9]}$. Obviously, the combined regimen of ceftriaxone and netilmicin is much more convenient to administer than penicillin plus streptomycin and it may also be less toxic ${ }^{\text {99. }}$. However, because of the limited experience of once-daily administration of aminoglycoside in endocarditis in humans, this cannot yet be recommended as a standard treatment. Regarding efficacy, streptomycin and gentamicin are probably equivalent ${ }^{[1]}$. Nowadays, gentamicin is generally preferred because it is better tolerated when administered intravenously and because serum levels can easily be monitored (Table 3 ).

Unless clearly indicated (see below), regimens including an aminoglycoside should probably not be used in patients with impaired renal or auditory function, or in older

Table 2 Suggested daily dosages for the major antibiotics used in streptococcal and enterococcal endocarditis

\begin{tabular}{|c|c|}
\hline $\begin{array}{l}\text { Penicillin G } \\
\text { Ampicillin } \\
\text { Ceftriaxone* } \\
\text { Vancomycin } \\
\text { Gentamicin } \\
\text { Streptomycin }\end{array}$ & $\begin{array}{l}6 \times 2-3 \text { million units } \\
6 \times 2 \mathrm{~g} \\
2 \mathrm{~g} \\
2 \times 15 \mathrm{mg} \cdot \mathrm{kg}^{-1}(>60 \mathrm{~min}) \\
3 \mathrm{mg} \cdot \mathrm{kg}^{-1}(\text { divided in } 2-3(17) \text { doses })_{2 \times 7.5 \mathrm{mg} \cdot \mathrm{kg}^{-1}(60 \mathrm{~min})}\end{array}$ \\
\hline
\end{tabular}

* Non-enterococcal streptococci only.
Table 3 Which aminoglycoside in streptococcal and enterococcal endocardutis?

Published clinical experience

Synergy in vitro and in animal models High level resistance in streptococci (rare)

High level resistance in enterococci

Tolerance to intravenous administration

Monitoring

\begin{tabular}{|c|c|c|}
\hline SM & $>$ & GN \\
\hline SM & $=$ & GM \\
\hline SM & $\gg$ & GN \\
\hline SM & $\gg$ & GN \\
\hline GM & $>$ & SM \\
\hline GM & $>$ & SI \\
\hline
\end{tabular}

SM = streptomycin; $\mathrm{GM}=$ gentamicin .

patients ${ }^{[18,9]}$. In these patients, sole administration of a beta-lactam for 4 weeks is preferred.

\section{Vancomycin or teicoplanin for 4 weeks}

In patients allergic to penicillin, ceftriaxone can be used if hypersensitivity is not of the immediate-type. In any patient with immediate-type hypersensitivity to penicillin or other beta-lactams, vancomycin can be used for 4 weeks at a dose of $15 \mathrm{mg} \cdot \mathrm{kg}^{-1}$ every $12 \mathrm{~h}$ (administered intravenously in over an hour) ${ }^{[1]}$. Serum levels should be monitored, especially in patients with renal dysfunction ${ }^{[9]}$. However, there is limited published experience with this agent for that indication. Teicoplanin, a glycopeptide with a long half-life allowing once daily administration, has also been used in viridans streptococcal endocarditis. However, of 10 patients who completed a 4-week course of teicoplanin ( $500 \mathrm{mg}$ every $12 \mathrm{~h}$ on the first 2 days and then $10 \mathrm{mg} . \mathrm{kg}^{-1}$ every $24 \mathrm{~h}$ ), nine were cured but one relapsed. Moreover, among the 20 patients who entered the study, five $(25 \%)$ experienced drug fever which prompted interruption of treatment ${ }^{[10]}$. Thus, teicoplanin deserves further investigation before it can be recommended as an alternative once-daily parenteral regimen for the treatment of streptococcal endocarditis. 


\section{Combined parenteral and oral regimens}

Although oral therapy with high dose oral amoxicillin or ampicillin plus probenecid has been successful in the treatment of streptococcal endocarditis, it is not generally recommended because serum levels are lower and less predictable than after parenteral administration ${ }^{[1]}$. Moreover, compliance may be a problem in some patients. However, combined parenteral and oral regimens have been successfully used in some countries ${ }^{[6]}$. Thus, Stamboulian et al. successfully treated 15 patients with 14 days of ceftriaxone followed by 14 days of high-dose oral amoxicillin. In these patients, serum bactericidal titres above $1 / 8$ was demonstrated while on oral treatment.

\section{Options for outpatient therapy}

Economic constraints have encouraged the treatment of endocarditis in the home ${ }^{[11]}$. This has been made feasible with the use of once-daily administration of ceftriaxone. In one study, among 55 patients treated for 4 weeks with this agent, 23 could be discharged from hospital after less than 2 weeks and seven, who had to stay in hospital for the entire course of treatment, only did so because of an inappropriate outpatient setting ${ }^{[2]}$. In a study performed in Argentina, seven out of 30 patients could be treated entirely as outpatients and 27 received both inpatient and outpatient treatment ${ }^{[6]}$.

Obviously, outpatient therapy should only be considered in the patient whose condition is stable and for whom medical supervision can be maintained ${ }^{[11}$, but treatment should probably be initiated in hospital. Patients who are haemodynamically unstable, who have large vegetations or other echocardiographic findings associated with high rates of complications, or who are suspected of distant metastatic complications, should stay in the hospital.

\section{Viridans streptococci: special situations}

In several situations, a 4-week course of a cell-wall active agent combined with an aminoglycoside for $2-4$ weeks is indicated ${ }^{[1.12]}$. These situations include nutritionally deficient variants of viridans streptococci, streptococci with a minimum inhibitory concentration for penicillin greater than $0.1 \mathrm{mg} . \mathrm{l}^{-1}$ but less than $0.5 \mathrm{mg} . \mathrm{l}^{-1}$, patients with extracardiac infection such as mycotic aneurysm, patients whose symptoms have lasted more than 3 months, patients who have experienced a relapse with the same organism and patients who are receiving penicillin prophylaxis for rheumatic fever. Some authorities also recommend such a regimen for prosthetic valve endocarditis ${ }^{[12]}$. In the published experience ${ }^{[12]}$, the cell-wall active agent most commonly used was penicillin $G$, but vancomycin has been recommended as an alternative. However, there is no reason why ceftriaxone or cefazolin would not work in these situations. The choice of the aminoglycoside has been discussed above.

\section{S. pneumoniae, group A streptococci}

Until recently, all strains of $S$ pneumoniae and group A streptococci were highly sensitive to penicillin. For these two microorganisms, penicillin $G$ and vancomycin (as an alternative in penicillin-allergic patients) have been rec- ommended ${ }^{[12]}$. Since these microorganisms are also extremely sensitive to ceftriaxone, this agent can probably also be used. However, due to the increasing incidence of $S$. pneumoniae resistant to penicillin in some parts of the world, careful testing of susceptibility should always be performed.

\section{Group B, C, G streptococci}

Group B streptococci are less sensitive to penicillin $G$ than are group $A$ isolates. Synergy between penicillin and an aminoglycoside has been demonstrated in-vitro against non-tolerant as well as tolerant isolates and in animal models of endocarditis. Therefore, a combination of cell-wall active agents combined with an aminoglycoside should be considered in these patients ${ }^{[12]}$. The same holds true for group $\mathrm{C}$ and group $\mathrm{G}$ streptococci ${ }^{[12]}$.

\section{Enterococci}

Enterococci are streptococci which belong to Lancefield's group D. They include members of several different streptococcal species, such as Enterococcus faecalis, Enterococcus faecium and Enterococcus durans. Treatment of enterococcal endocarditis is complicated, because these microorganisms are more resistant to penicillin $\mathrm{G}$ (median

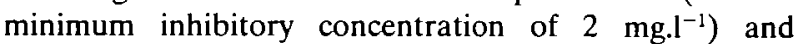
to extended spectrum penicillins than most other streptococci; they are also uniformally resistant to cephalosporins $^{[3]}$. In addition, cell-wall active antibiotics are only bacteriostatic ${ }^{[1]}$. Although enterococci are also resistant to aminoglycoside antibiotics, the combination of penicillin, ampicillin, or vancomycin with certain aminoglycoside antibiotics has been found to exercise a synergistic bactericidal effect on these bacteria, provided that they do not display a high level of resistance ${ }^{[13]}$. A few years ago, the problem became much more complicated because of the emergence of strains resistant to one or more of the following classes of antibiotics: beta-lactams, glycopeptides and aminoglycosides (Table 4).

Regarding beta-lactams, the resistance can be caused either by decreased affinity of penicillin-binding proteins to the drug (intrinsic resistance) or to beta-lactamase production $^{[3,14]}$. Recently, strains resistant to vancomycin and/or teicoplanin have also been identified. The mechanism of resistance has not yet been fully characterized, but several phenotypes (Van A, Van B, Van C) ranging from a low to a high-level of resistance, have been described ${ }^{[15]}$.

The degree of resistance to aminoglycosides is highly variable. A minimum inhibitory concentration greater than or equal to $2000 \mathrm{mg} . \mathrm{l}^{-1}$ is generally considered to be the limit between a low and a high-level of resistance ${ }^{[1]}$. Enterococci which are highly resistant to an aminoglycoside are not killed synergistically when this agent is combined with a cell-wall active agent in-vitro or in experimental models ${ }^{[1.16]}$. Resistance is due to the presence of various enzymes which modify the structure of the aminoglycoside ${ }^{[3]}$. Since the enzymes involved in the resistance to streptomycin and gentamicin are different, some strains resistant to one of the aminoglycosides might be susceptible to the other. 
Table 4 Enterococci: resistance $(R)$ to antibiotics

Beta-lactams

(a) Intrinsic resistance (PBPs)

(b) Beta-lactamase (plasmids)

Glycopeptides

(a) Van $\mathrm{C}$

(b) Van B

low level $\mathrm{R}$ to vancomycin

intermediate level $R$ to vancomycin

(c) $\operatorname{Van} A$ susceptible to teicoplanin

resistant to teicoplanin

Aminoglycosides

(a) high level $\mathrm{R}$ to streptomycin

(b) high level $\mathrm{R}$ to gentamicin

(c) high level $\mathrm{R}$ to gentamicin

low level $R$ to gentamicin

low level $\mathrm{R}$ to streptomycin

high level $\mathrm{R}$ to streptomycin

$\mathrm{R}=$ resistance; $\mathrm{PBP}=$ penicillin binding protein.

A suggested strategy for the choice of treatment of enterococcal endocarditis is outlined in Table 5. It should be kept in mind that combined therapy is required for successful treatment: treatment with penicillin alone has resulted in relapse rates of $25 \%$ or more. The minimum duration of treatment should be 4 weeks but many authorities suggest 6 weeks ${ }^{[1]}$. The first step is to choose a cell-wall active agent. If the strain is sensitive to ampicillin, this agent should be chosen. Since low levels of beta-lactamase production might not be detected by routine susceptibility testing, beta-lactamase production should be looked for if ampicillin is used ${ }^{[13,14]}$. In some experimental studies, continuous intravenous infusion was found to be more effective than intermittent dosing ${ }^{[16]}$. If a strain produces beta-lactamase, amoxicillin/clavulanate can probably be substituted, although there are no data on the clinical efficacy of this association in this indication. If the strain is intrinsically resistant to beta-lactams, vancomycin should be used unless the strain is also resistant to this antibiotic.
Some strains resistant to vancomycin have retained susceptibility to teicoplanin ${ }^{[3]}$. If the strain is resistant to both beta-lactams and glycopeptides, a triple combination of vancomycin, penicillin or ampicillin and aminoglycoside can be tried, provided that the strain is not highly resistant to the aminoglycoside ${ }^{[13]}$. Hence, vancomycin has been shown to reduce the minimum inhibitory concentration to penicillin when used with other drugs.

If the strain is not highly resistant to streptomycin or gentamicin, the synergistic effect provided by these two agents is similar ${ }^{[1]}$. Other aminoglycosides cannot routinely be substituted. If the strain is highly resistant to gentamicin, streptomycin should be tested. If high levels of resistance are present for both agents, the synergistic effect is lost and the aminoglycoside should therefore not be used.

Despite encouraging reports adding ciprofloxacin or rifampicin when dealing with multi-resistant strains provides no definite benefit ${ }^{[3]}$. However, recently, the addition of chloramphenicol or tetracycline has been found to be

Table 5 Enterococci: therapeutic options

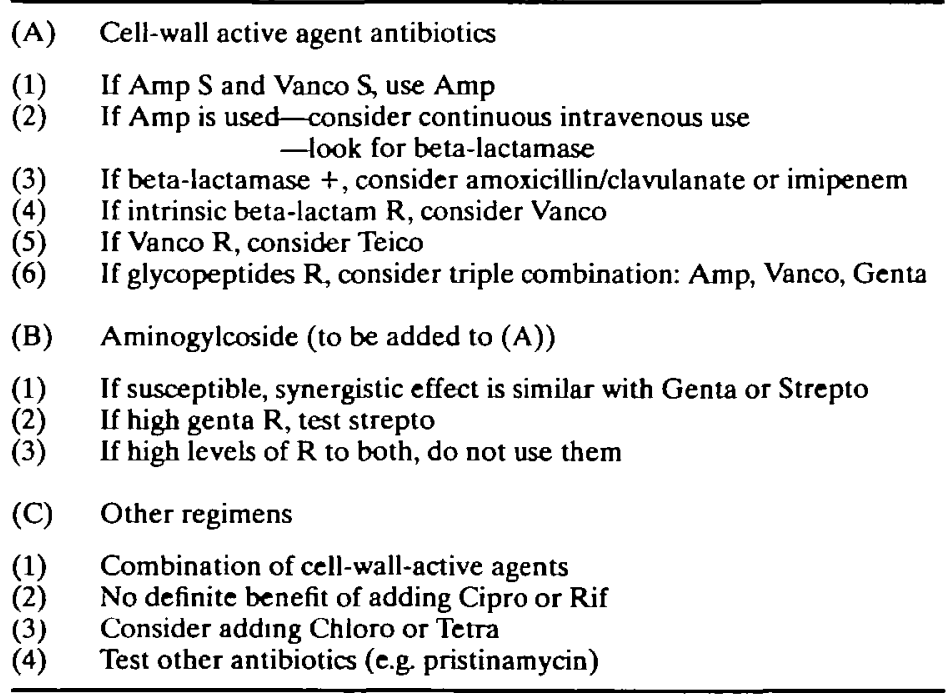

Amp = ampicillin; Vanco = vancomycin; Teico = teicoplanin $;$ Cipro $=$ ciproxin; Rif = rifampicin; Chloro = chloramphenicol; Genta = gentamicin; Strepto = streptomycin; Tetra = tetracycline; $S=$ sensitive; $R=$ resistant. 
useful in some situations. It should be mentioned that trimethoprim sulfamethoxazole appears to have an inhibitory effect against many strains of enterococci when tested in vitro. However, this agent is probably ineffective in-vivo ${ }^{[3]}$.

Despite some success with medical therapy alone in endocarditis due to multi-resistant strains of enterococci, surgical removal of the infected valve is often the only curative treatment.

\section{References}

[1] Bisno AL, Dismukes WE, Durack DT et al. Antimicrobial treatment of infective endocarditis due to viridans streptococci, enterococci, and staphylococci. JAMA 1989; 261: 1471-7.

[2] Francioli P, Etienne J, Hoigne R, Thys JP, Gerber A. Treatment of streptococcal endocarditis with a single daily dose of ceftriaxone for 4 weeks: Efficacy and out-patient treatment feasibility. JAMA 1992; 267: 264-7.

[3] Eliopoulos GM. Aminoglycoside resistant enterococcal endocarditis. In: Wilson WR, Steckelberg JM, eds. Infectious Disease Clinics of North America. Philadelphia: W. B. Saunders Company, 1993: 117-33.

[4] Wilson WR, Geraci JE. Treatment of streptococcal infective endocarditis. Am J Med 1985; 78(Suppl 6B): 128-13.

[5] Durack DT. Review of early experience in treatment of bacterial endocarditıs, 1940-1955. In: Bisno AL, ed. Treatment of infective Endocarditis. New York: Grune \& Stratton, 1981: 1-14.

[6] Stamboulian D, Bonvehi P, Arevalo $C$ et al Antibiotic management of outpatients with endocarditis due to penicillinsusceptible streptococci. RID 1991; 13(Suppl 2): S160-3.

[7] Wilson WR. Ceftriaxone sodium therapy of penicillin G-susceptible streptococcal endocarditis. JAMA 1992; 267: $279-80$.
[8] Wilson WR, Thompson RL, Wilkowske CJ. Short-term therapy for streptococcal infective endocarditis. Combined intramuscular administration of penicillin and streptomycin. JAMA $1981 ; 245:$ 360-3.

[9] Stamboulian D, Ruch W, Francioli P. Treatment of streptococcal endocarditis with a single dose of ceftriaxone + netilmicin for 14 days. ESCMID, Sevilla, 1993; (Abstract No 1104, p. 254).

[10] Venditti M, Gelfusa V, Serra P, Brandimarte C, Micozzi A, Martino P. 4-week treatment of streptococcal native valve endocarditis with high-dose teichoplanin. Antimicrob Agents Chemother 1992; 36: 723-6.

[11] Francioli PB. Ceftriaxone and outpatient treatment of infective endocarditis. In: Wilson WR, Steckelberg JM, eds. Infectious disease clinics of North America. Philadelphia: W. B. Saunders Company, 1993: 97-115.

[12] Roberts RB. Streptococcal endocarditis: the viridans and beta hemolytic streptococci. In: Donald $K$, ed. Infective Endocarditis, 2nd edn. New York: Raven Press, 1992: 191-208.

[13] Caron F, Carbon C, Gutmann L. Triple-combination penicillin-vancomycin-gentamicin for experimental endocarditis caused by a moderately penicillin- and highly glycopeptideresistant isolate of enterococcus faecium. J Infect Dis 1991; 164: 888-93.

[14] Goudron PE, Markowitz SM, Wong ES. Isolation of a betalactamase-producing, amynoglycoside-resistant strain of enterococcus faecium. Antimicrob Agents Chemother 1992; 36: 1125-6.

[15] Derlot E, Courvalin P. Mechanisms and implications of glycopeptide resistance in enterococci. Am J Med 1991; 91(Suppl 3B): 82S-85S.

[16] Cercenado E, Eliopoulos GM, Wennersten C, Moellering $\mathrm{RC} \mathrm{J}_{\mathrm{r}}$. Influence of high-level gentamicin resistance and beta-hemolysis on susceptibility of enterococci to the bacterial activities of ampicillin and vancomycin. Antimicrob Agents Chemother 1992; 36: 2526-8. 